\title{
Prediabetes: Challenges, Novel Solutions, and Future Directions
}

\author{
Authors: \\ *Monique E Francois, ${ }^{1,2}$ Katie M Oetsch ${ }^{1,2}$ \\ 1. School of Medicine, University of Wollongong, Australia \\ 2. Illawarra Health and Medical Research Institute, Wollongong, Australia \\ *Correspondence to francois@uow.edu.au \\ Disclosure: $\quad$ The authors have declared no conflicts of interest. \\ Received: \\ 05.07.21 \\ Accepted: \\ 06.10 .21 \\ Keywords: \\ Exercise, hyperglycaemia, impaired glucose tolerance, lifestyle, nutrition, weight loss. \\ Citation: \\ EMJ. 2022; DOI/10.33590/emj/21-00148.
}

\section{Abstract}

Prediabetes: Prediabetes is a salient state of hyperglycaemia and/or hyperinsulinaemia that often precedes a diagnosis of Type 2 diabetes (T2D). It is projected that by 2040 , almost $8 \%$ of the global population will have prediabetes, with approximately $70 \%$ progressing to T2D within their lifetime. Abnormal glucose tolerance increases the risk of associated complications, including cardiovascular disease, stroke, and microvascular diseases, all of which are major contributors to the global healthcare burden. T2D alone is predicted to cost the healthcare system upwards of 490 billion USD by 2030 , thus addressing this growing burden is vital.

Challenge One. Diagnosis and classification: Diagnosis poses a challenge and there is debate between leading world expert panels regarding thresholds, notably between the World Health Organization (WHO) and American Diabetes Association (ADA) for impaired fasting glucose. Hyperinsulinaemia may also go undetected as this is not currently routinely tested or used as diagnostic criteria. This has been largely due to cost and lack of consensus data for appropriate diagnostic threshold; however, with disease burden costs estimated to be close to half a billion USD by the end of the decade, an in-depth cost analysis for benefits-costs of early detection and treatment or prevention is warranted.

Challenge Two. Health messaging and public perception: Prediabetes can revert to normoglycaemia with diet and lifestyle interventions. This, however, is not conveyed well in public health messaging. In addition to public perception about the likelihood of disease progression to T2D, prediabetes is not considered a disease state, which may also influence public perception regarding perceived urgency of treatment and necessity for intervention.

Challenge Three. Intervention and treatment: Diet and lifestyle interventions are heralded as best practice when it comes to prediabetes management, and metformin for those at greatest risk of future T2D. Synergistic use of the available novel and promising interventions including low carbohydrate diets, higher protein diets, time restricted feeding, and high intensity interval training may help time-poor individuals achieve improvements in risk-factors including weight loss and glycaemic control (HbA1c and fasting plasma glucose). As large-scale feasibility and adherence are major obstacles to contend with in the rollout of diet and lifestyle interventions, personalised approaches, coupled with counselling based on social cognitive theory, may be increasingly utilised to target specific groups and individuals as programmes can be tailored to meet specific needs and preferences. 


\section{SHOULD PREDIABETES BE TAKEN MORE SERIOUSLY?}

\section{Diagnosis}

Prediabetes is a salient state of hyperglycaemia and/or hyperinsulinaemia that persists for years, often preceding a diagnosis of Type 2 diabetes (T2D). The diagnostic criteria for prediabetes is difficult to define and still debated. The American Diabetes Association (ADA) and the World Health Organisation (WHO) agree on a diagnosis for impaired glucose tolerance (IGT) and HbA1c but differ in their criteria for impaired fasting glucose (IFG). Both entities consider a 2-hour $75 \mathrm{~g}$ Oral Glucose Tolerance Test (OGTT) result $7.8-11.0 \mathrm{mmol} / \mathrm{L}(140-199 \mathrm{mg} / \mathrm{dL})$ or $\mathrm{HbA1c}$ $5.7-6.4 \% \quad(39-47 \mathrm{mmol} / \mathrm{mol})$ to diagnose prediabetes. The diagnosis for IFG varies, with the ADA using a lower threshold of 5.6-6.9 mmol/L (100-125 mg/dL) compared to the WHO using a higher threshold of 6.1-6.9 $\mathrm{mmol} / \mathrm{L}(110-125 \mathrm{mg} / \mathrm{dL})$. While in theory, a lower threshold should provide earlier detection and an increased window for prevention, research has indicated that the lower threshold does not mirror actual risk for T2D development, ${ }^{1}$ nor may it provide significant gain in T2D prevention. ${ }^{2}$ The higher threshold appears a more accurate indicator of disease progression. ${ }^{3}$

\section{Disease Classification}

Prediabetes can also be classified or termed 'intermediate hyperglycaemia', which is the preferred terminology of the WHO. ${ }^{3}$ The term 'prediabetes' indicates a pre-disease state, whereas 'intermediate hyperglycaemia' alludes to a mild disease and one that has independent consequences of a T2D diagnosis. Although at present prediabetes is not considered a mild disease, the shift towards acceptance as an independent pathological entity is supported by inclusion in the 2015 International Statistical Classification of Diseases and Related Health Problems (ICD). ${ }^{4}$ This inclusion is important for public perception as it conveys the medically supported need for treatment or intervention. Treatments need not be pharmaceutical. Treatment and prevention should instead take the form of diet and exercise lifestyle intervention by allied health professionals. ${ }^{5}$ These pathways reduce the over medicalisation and unsustainable burden for healthcare systems. This paper acknowledges the importance of treating intermediate hyperglycaemia an independent state but will use the more commonly accepted term 'prediabetes' to minimise confusion.

\section{Consequences}

Prediabetes has far-reaching complications, with approximately 25\% developing T2D within 3-5 years $^{6}$ and $70 \%$ progressing within their lifetime. ${ }^{7}$ In 2010, the estimated global expenditure for T2D was 376 billion USD $^{8}$ and is expected to rise beyond 490 billion USD by $2030 .{ }^{8}$ In 2015 it was estimated that $6.7 \%$ of the world's population had prediabetes, with an estimate of $7.8 \%$ projected by $2040 .{ }^{9}$ The predictions for prediabetes are significantly higher (35-39\%) in regions such as Catalonia, ${ }^{10}$ China, ${ }^{11}$ and the USA. ${ }^{12}$ Furthermore, according to the Centers for Disease Control and Prevention (CDC [2020]) 84\% of cases are undiagnosed. Similar numbers are seen in Australia as one in four adults are thought to be living with undiagnosed prediabetes/T2D.13 As those with prediabetes are at increased risk of cardiovascular disease (CVD), stroke, and microvascular diseases, including retinopathy, nephropathy, and neuropathy, ${ }^{14}$ diagnosing and treating abnormal glucose tolerance early in the diagnostic process is essential to minimise the future burden of disease.

A prediabetes diagnosis does not guarantee disease progression. Approximately $5-10 \%$ of those diagnosed revert back to normoglycaemia each year ${ }^{6}$ and this may be spontaneous. ${ }^{15}$ A recent cohort study found that participants who returned to normoglycaemia after a IFG diagnosis were younger and had lower BMI. ${ }^{10}$ As BMl is a modifiable risk factor, interventions focusing on diet and exercise for weight loss have been the focus of research; however, pharmaceutical trials have also been undertaken with varying success.

\section{WEIGHT LOSS AND THE ROLE OF DIET AND EXERCISE INTERVENTIONS IN PREDIABETES MANAGEMENT}

\section{Findings from the DQDPS}

The DQDPS is a 6-year lifestyle intervention in China that saw 576 patients with prediabetes across 33 clinics randomised into one of four 
intervention or control groups. ${ }^{16}$ The researchers compared changes in OGTT and fasting plasma glucose between those randomised to diet only, exercise only, or the combination of exercise and diet intervention. After 6 years, 32\% had returned to normoglycaemia, 21\% remained with IGT, ${ }^{17}$ and $47 \%$ progressed to T2D..$^{7}$ The majority (68\%) who progressed to T2D were from the control, with progression $25 \%$ lower in the interventions. ${ }^{16}$ Those who progressed to T2D had higher baseline BMI and fasting plasma glucose when compared with those who remained IGT or returned to normoglycaemia. ${ }^{17}$

Five hundred and forty participants were followed-up for 24 years, making this a 30-year landmark study for the long-term effects of diet and exercise intervention in prediabetes. At the 24-year follow-up, those who remined with IGT had a median delay for T2D onset of 10 years, a 34\% lower incidence of CVD, and 52\% lower incidence of microvascular disease when compared to those who progressed to T2D during the 6-year trial. Those who reverted to normoglycaemia during the trial had a median delay for T2D onset of 15 years, a 37\% lower incidence of CVD, and $66 \%$ lower incidence of microvascular disease. ${ }^{17}$ These findings demonstrate that diet and exercise interventions have significant long-term effects not only on T2D progression but also on associated risk factors and complications. As a lower BMI had favourable outcomes, diet and exercise interventions with a weight loss focus are likely most beneficial.

\section{Weight Loss}

Obesity is a key risk factor in the development of prediabetes and T2D. Weight loss of $5-10 \%$ can reduce 3 -year incidence of T2D by $60 \% 18$ and, thus, is a clinically relevant target for diet and exercise interventions. Low engagement ${ }^{19}$ and difficulty maintaining lifestyle changes ${ }^{20}$ are often reported as major barriers to successful long-term behaviour change and weight loss. While many traditional dietary recommendations feature low-fat and calorie restriction, ${ }^{21}$ the ADA care guidelines were updated in 2019 to include low carbohydrate diets as another strategy to improve glycaemia. ${ }^{20}$

\section{Low-Carbohydrate Diets}

Low-carbohydrate diets have gained recent traction due to promising results for weight loss, improved HbA1c, and remission for both prediabetes and T2D.22 Additionally, despite some negative publicity concerning the safety of low-carbohydrate diets, a recent meta-analysis ${ }^{22}$ reported no statistically significant or clinically important detrimental markers for CVD, including lipid profile or C-reactive protein when compared to low-fat calorie restricted diets. While the benefit of low carbohydrate diets for glucose control are largely attributed to the reduction in foods that raise blood glucose levels, consequently reducing insulin demands, another advantage of low carbohydrate diets over traditional dietary recommendations is their ability to increase feelings of satiety by reducing the hunger hormones insulin and ghrelin and increasing satiety hormones glucagon-like peptide 1 and peptide tyrosine tyrosine. ${ }^{23}$ For example, when a low-fat, calorie-restricted diet containing $150 \mathrm{~g}$ of carbohydrate was compared to a low-carbohydrate, unrestricted-calorie diet containing less than $50 \mathrm{~g}$ net carbohydrate, it was found that the total calories consumed between the groups throughout the 3 month study were almost identical, ${ }^{21}$ suggesting that the low-carbohydrate group naturally reduced their calorie intake. Interestingly, while consuming a similar energy intake, the low-carbohydrate group saw a mean weight loss of almost $6 \mathrm{~kg}$, more than double the traditional treatment group. Additionally, this study also found 50\% of low carbohydrate participants had a clinically significant decrease of $0.5 \%$ in their $\mathrm{HbA1c}$, compared to $22.0 \%$ in the traditional dietary treatment group.

A 2020 pilot study ${ }^{19}$ looked at adapting the CDC's National Diabetes Prevention Program (NDDP) to a low-carbohydrate diet-DDP (LC-DDP). The NDDP is an American lifestyle intervention programme designed to be affordable and accessible. The NDDP currently has a real-world success rate of $35 \%$ of participants meeting their weight loss goal of $5 \%$. The LC-DDP pilot maintained the same delivery format as the NDDP, changing only the dietary advice from low-fat calorie restriction to a net carbohydrate intake of 20-25 g daily. This dietary change saw $50 \%$ of participants reach $>5 \%$ weight loss after 6 months while reporting both diet enjoyment 
and diminished cravings. After 12 months, 71\% of participants reported still adhering to the low carbohydrate approach. The success of the LC-DDP indicates that low-carbohydrate diets are not only successful at achieving weight loss in the short term but are also acceptable and manageable long-term. Whilst a large proportion of the carbohydrates are replaced by fats, many low-carbohydrate diets also naturally increase protein as part of the macronutrient redistribution.

\section{High Protein Diets}

High-protein diets may also suppress hunger, increase satiety, and therefore assist in weight loss. ${ }^{24}$ The satiating effects are achieved through the gut-endocrine axis as protein consumption reduces ghrelin and increases peptide tyrosine tyrosine..$^{25,26}$ Recently, Drummen et al. ${ }^{27}$ found that a high-protein diet (25\% protein) promoted initial weight loss and prevented weight regain over a 3-year follow-up period. ${ }^{27}$

A high protein diet (30\% protein) followed for 6 -months resulted in $100 \%$ reversal to normoglycaemia in people with prediabetes, compared with $33 \%$ in control. ${ }^{24}$ Significant improvements in insulin sensitivity were also seen in the high protein group, as well as reduced CVD risk, oxidative stress, and inflammatory cytokines. In agreement, a recent systematic review reported improvements in insulin sensitivity and lipid profiles with a high protein diet across 13 studies in T2D. ${ }^{28}$ Therefore, increasing protein and lowing carbohydrates is beneficial for weight loss and glycaemic control; however, meal timing may also be an important factor to consider.

\section{Time Restricted Feeding and Intermittent Fasting}

While very low-carbohydrate diets can reinforce circadian rhythm and reduce oxidative stress and inflammation, ${ }^{29}$ these benefits are in line with 'metabolic fasting'. Actual fasting through reduced eating windows and increased time between meals has been shown to have similar metabolic effects. ${ }^{29}$ Fasts need not be long, and it appears meal composition and calorie content has minimal bearing on results. ${ }^{29}$ In a tightly controlled study, Peeke et al. ${ }^{29}$ provided identical calorie-controlled meals through the Jenny Craig Rapid Results programme to intervention and control groups for 8 weeks. The intervention group were instructed to fast for 14 hours (a pattern of 14:10), while the control group fasted for 12 hours (12:12). A statistically significant difference of $2 \mathrm{~kg}$ in weight loss was found between the groups simply by increasing the fasting window by 2 hours (-11 kg intervention versus $-9 \mathrm{~kg}$ control). Another study found that time restricted feeding early in the day (6-hour eating window $<3$ p.m.) improved insulin sensitivity, $\beta$ cell responsiveness, blood pressure, and oxidative stress in people with prediabetes when compared to a fasting pattern of 12:12.30 Results from the above studies were intentionally independent of weight loss, demonstrating the benefits of intermittent fasting that extend beyond just weight loss.

\section{Exercise}

Exercise is considered a cornerstone therapy for weight loss and the management of lifestyle diseases such as prediabetes. ${ }^{31}$ Exercise also provides benefits beyond weight loss, and overwhelming evidence suggests this is independent of exercise type. ${ }^{32}$ Many regulatory bodies agree that $150 \mathrm{~min}$ of moderate to vigorous exercise per week is adequate; 33 however, $35 \%$ of those from more affluent countries do not meet this weekly requirement. ${ }^{32}$ Often, 'lack of time' is cited as a key hurdle to maintaining a regular exercise pattern. ${ }^{32}$ Recently research has experienced a shift towards exercise patterns that produce favourable outcomes while reducing the time burden. High intensity interval training (HIIT) is one way to achieve this.

\section{High Intensity Interval Training}

HIIT refers to short bursts of high intensity exercise interspersed with low intensity recovery or rest periods. A typical goal is to maintain approximately $90 \%$ of maximal effort (heart rate max) for a total of $10-15$ min with periodised rests between exercise bursts. ${ }^{33}$ A recent metaanalysis found HIIT increased cardiorespiratory fitness more than moderate intensity continuous training (MICT); however, both similarly improve HbA1c, blood pressure, lipid profile, BMI, or hip-to-waist ratio; ${ }^{34}$ suggesting that similar health outcomes can be achieved through HIIT and shorter exercise duration. A 12-week pilot study $^{35}$ in 35 participants with prediabetes found a training intervention that included two 
resistance/strength training sessions and either 30 min of HIIT or MICT for approximately 30 min 3 days per week, in line with the physical activity guidelines, similarly improved many of the above-mentioned markers of metabolic health.

The benefit of HIIT for long-term adoption is that there are countless ways it can be tailored to suit the individual. Usually cumulative intervals $\leq 15 \mathrm{~min}$ are considered low volume HIIT (LV-HIIT) and cumulative intervals $\geq 15 \mathrm{~min}$ are high volume HIIT (HV-HIIT). ${ }^{32}$ A recent 12-week study in 77 participants who were overweight with prediabetes demonstrated a significant reduction with HV-HIIT and LV-HIIT for HbA1c of $1.27 \%$ and $0.87 \%$, and fasting blood glucose of $0.9 \mathrm{mmol} / \mathrm{L}$ and $0.7 \mathrm{mmol} / \mathrm{L}$, respectively. ${ }^{33}$ Of interest, the HV-HIIT protocol was almost double the length of the LV-HIIT protocol (4O min versus $25 \mathrm{~min}$ ). These findings of greater improvements; however, are consistent with a recent topical review suggesting that $\mathrm{HV}-\mathrm{HIIT} \geq \mathrm{LV}-\mathrm{HIIT} \geq \mathrm{MICT}$ across most measurable outcomes. ${ }^{32}$ As the higher volume typically requires longer duration intervals (i.e., $4 \mathrm{~min}$ ) this is not always a viable option for those with time constraints. The efficacy of LV-HIIT (i.e., $1 \mathrm{~min}$ intervals) must, therefore, be compared to that of HV-HIIT in the interest of maximising results in a shorter period.

The cardiometabolic benefits of exercise are conditional on continued engagement and participation for ongoing results. ${ }^{31}$ Engagement in MICT is typically low ${ }^{31}$ and while no studies have reported the efficacy of HIIT past 1 year, there is promising data that HIIT may be more sustainable than MICT in adults with prediabetes. ${ }^{36}$ A recent study was conducted combining the use of counselling strategies based on social cognitive theory with either MCIT or HIIT. ${ }^{37}$ After 1 year follow-up, females who received the counselling in self-regulatory techniques such as goal-setting, planning, and self-monitoring reported higher levels of continued exercise engagement. ${ }^{38}$

The authors know that both diet and exercise combined are essential elements of disease prevention. Whilst combining HIIT with a low-carbohydrate diet has been postulated, ${ }^{39}$ there are yet to be studies combining the two strategies. It is hypothesised that combining these strategies will synergistically maximise the benefits of both approaches ${ }^{39}$ and may prove to be an optimal diet and exercise combination for prediabetes and T2D.

\section{PHARMACEUTICAL INTERVENTIONS FOR PRE-DIABETES}

Pharmaceutical trials in prediabetes have had variable success across a range of new medications and those already approved for $T 2 D$ management. ${ }^{6,40,41}$ Pharmaceutical interventions for prediabetes are not currently routine based on limited efficacy, side effects, and/or cost. ${ }^{42}$ Due to its relative low cost and safety, metformin is the exception and may be considered for those with a BMI of $>35 \mathrm{~kg} / \mathrm{m}^{2}$, diagnosed $<60$ years, and previous gestational diabetes. ${ }^{42}$ It is worth noting that unlike lifestyle changes such as exercise, pharmaceutical interventions do not demonstrate meaningful effects on many key outcomes for cardiovascular events or mortality, ${ }^{40}$ including improvement in body composition or arterial stiffness.

Lifestyle modifications through diet and exercise remain the most effective preventive strategies for prediabetes; ${ }^{40}$ however, they are often criticised as resource intensive and unsustainable. Pharmaceutical interventions are often cited as a viable inexpensive alternative, ${ }^{40}$ yet lifestyle interventions are more cost effective than even metformin. Therefore, diet and lifestyle interventions remain the safest and most cost-effective treatment for prediabetes.

\section{FUTURE DIRECTIONS}

\section{Diagnosis and Intervention}

At least half of all prediabetes cases are thought to be undiagnosed. ${ }^{13,43}$ Given early intervention can prevent ensuing disease, developing alternate methods to address prediabetes detection is paramount. One cost effective measure may be to increase the administration of existing T2D screening tools. A recent study ${ }^{44}$ trialled pre-screening using the Finnish Diabetes Risk Score Calculator (FINDRISC) questionnaire as part of World Diabetes Day awareness. Of the 3,351 questionnaires administered to those without previously known prediabetes or T2D, as many as $15 \%$ reported a high-risk score $(n=420)$. Laboratory diagnostics for HbA1c and fasting blood glucose 
were administered to 397 of these and 40 subjects were diagnosed with T2D. Prediabetes was found in 213 subjects; more than half of those who returned a high-risk score on the pre-screening questionnaire.

Alternatively, the measurement of insulin resistance in diagnostic criteria may also be considered. One difficulty with prediabetes diagnosis is that hyperinsulinaemia can mask abnormal glucose levels for years. ${ }^{45}$ Although more expensive, measuring plasma insulin is a much more accurate method of identifying insulin dysregulation and associated T2D risk. Increasingly, novel approaches such as plasma amino acid profiles and specific microRNA biomarkers are also emerging as possible future diagnostic criteria; 46,47 however, these are still largely in developmental phase. Improving diagnostics is only part of the puzzle, as improving lifestyle intervention strategies and rollout are crucial to pre-diabetes management post-diagnosis.

As large-scale feasibility and adherence are major obstacles in the rollout of lifestyle interventions, ${ }^{37}$ personalised approaches may be increasingly utilised to target specific groups and individuals needs and preferences. For example, taking several elements from effective diet and exercise interventions such as the LC-DDP, or low-carbohydrate diet paired with tension and trauma release exercises and HIIT may be the most effective strategy for prediabetes. ${ }^{39}$ However, these require people to change several aspects of their daily routine and may not be suited for higher-risk individuals. ${ }^{32}$ The challenge may then be to find the minimum intervention required across each domain of diet and exercise to elicit beneficial results in either reversing prediabetes or delaying the onset of T2D. The establishment of a minimum benchmark would be beneficial for those who struggle with the long-term feasibility of more onerous intervention styles. By establishing recognised minimum requirements and best practice, allied health professionals could then work with individuals to create a tailored programme to best suit the needs of the individual. Effective short-term counselling based on social cognitive theory tailored to an individual's approach has the potential to promote long-term self-regulation and management, minimise ongoing financial barriers, and maximise independence. ${ }^{37}$ Finally, public health campaigns communicating the reversable nature of prediabetes are also warranted as there appears to be a lack of messaging around positive future disease outcomes in this space.

\section{References}

1. Forouhi NG et al. Incidence of type 2 diabetes in England and its association with baseline impaired fasting glucose: the Ely study 19902000. Diabet Med. 2007;24(2):200-7.

2. Lee CMY et al. Comparing different definitions of prediabetes with subsequent risk of diabetes: an individual participant data metaanalysis involving 76513 individuals and 8208 cases of incident diabetes. BMJ Open Diabetes Res Care. 2019;7(1):e000794.

3. World Health Organisation (WHO), International Diabetes Federation (IDF). Definition and diagnosis of diabetes mellitus and intermediate hyperglycemia: report of a WHO/IDF consultation Geneva, Switzerland. 2006. Available at: https://www. who.int/diabetes/publications/ Definition\%20and\%20diagnosis\%20 of\%20diabetes_new.pdf. Last accessed: 7 June 2021.

4. International Statistical Classification of Diseases and Related Health
Problems (ICD). ICD-10-CM code R73.03. Prediabetes. 2020. Available at: https://icdlist.com/icd-10/R73.03 Last accessed: 7 June 2021.

5. Diabetes Prevention Program (DPP) Research Group. 10-year follow-up of diabetes incidence and weight loss in the Diabetes Prevention Program Outcomes Study. Lancet. 2009;374(9702):1677-86.

6. Tabák AG et al. Prediabetes: a highrisk state for diabetes development. Lancet. 2012;379(9833):2279-90.

7. Hostalek U. Global epidemiology of prediabetes - present and future perspectives. Clinical Diabetes and Endocrinol. 2019;5:5.

8. Zhang $P$ et al. Global healthcare expenditure on diabetes for 2010 and 2030. Diabetes Res Clin Pract. 2010;87(3):293-301.

9. Ogurtsova $\mathrm{K}$ et al. IDF diabetes atlas: global estimates for the prevalence of diabetes for 2015 and 2040. Diabetes Res Clin Pract. 2017;128:40-50.
10. Falguera M et al. Prevalence of prediabetes and undiagnosed diabetes in the Mollerussa prospective observational cohort study in a semirural area of Catalonia. BMJ Open. 2020;10(1):e033332.

11. Wang $L$ et al. Prevalence and ethnic pattern of diabetes and prediabetes in China in 2013. JAMA. 2017;317(24):2515-23.

12. Reedy-Cooper A et al. Metformin to prevent diabetes in patients at increased risk. Am Fam Physician. 2020;102(9):531-2.

13. Dunstan DW et al. The rising prevalence of diabetes and impaired glucose tolerance. Diabetes Care. 2002;25(2):829-34.

14. Sami $W$ et al. Effect of diet on type 2 diabetes mellitus: a review. Int $J$ Health Sci (Qassim). 2017;11(2):65-71.

15. DeFronzo RA et al. Pioglitazone for diabetes prevention in impaired glucose tolerance. $\mathrm{N}$ Engl J Med. 2011;364:1104-15. 
16. Gong $Q$ et al. Morbidity and mortality after lifestyle intervention for people with impaired glucose tolerance: 30year results of the Da Qing Diabetes Prevention Outcome Study. Lancet Diabetes Endocrinol. 2019;7(6):45261.

17. Chen $Y$ et al. Associations of progression to diabetes and regression to normal glucose tolerance with development of cardiovascular and microvascular disease among people with impaired glucose tolerance: a secondary analysis of the 30 year Da Qing Diabetes Prevention Outcome Study. Diabetologia. 2021;64(6):1279-87.

18. Diabetes Prevention Program (DPP) Research Group. reduction in the incidence of Type 2 diabetes with lifestyle intervention or metformin. N Engl J Med. 2002;346(6):393-403.

19. Dina Hafez $\mathrm{G}$ et al. Mixed methods pilot study of a low-carbohydrate diabetes prevention programme among adults with pre-diabetes in the USA. BMJ Open. 2020;10(1):e033397.

20. Yost $O$ et al. Continuous glucose monitoring with low-carbohydrate diet coaching in adults with prediabetes: mixed methods pilot study. JMIR Diabetes. 2020;5(4):e21551.

21. Saslow $L R$ et al. A randomized pilot trial of a moderate carbohydrate diet compared to a very low carbohydrate diet in overweight or obese individuals with type 2 diabetes mellitus or prediabetes. PLoS One. 2014:9(4):e91027.

22. Goldenberg JZ et al. Efficacy and safety of low and very low carbohydrate diets for type 2 diabetes remission: systematic review and meta-analysis of published and unpublished randomized trial data. BMJ. 2021;372:m4743.

23. Chao AM et al. Dietary interventions for obesity: clinical and mechanistic findings. J Clin Invest. 2021;131(1):1-10.

24. Stentz FB et al. Remission of pre-diabetes to normal glucose tolerance in obese adults with high protein versus high carbohydrate diet: randomized control trial. BMJ Open Diabetes Res Care. 2016;4(1):e000258.

25. Batterham RL et al. Critical role for peptide $Y Y$ in protein-mediated satiation and body-weight regulation Cell Metab. 2006;4(3):223-33.
26. Blom WAM et al. Effect of a highprotein breakfast on the postprandial ghrelin response. Am J Clin Nutr. 2006;83(2):211-20.

27. Drummen $M$ et al. High compared with moderate protein intake reduces adaptive thermogenesis and induces a negative energy balance during long-term weight-loss maintenance in participants with prediabetes in the postobese state: a preview study. J Nutr. 2020;150(3):458-63.

28. Yu Z et al. Effects of high-protein diet on glycemic control, insulin resistance and blood pressure in type 2 diabetes: a systematic review and meta-analysis of randomized controlled trials. Clin Nutr. 2020;39(6):1724-34

29. Peeke PM et al. Effect of time restricted eating on body weight and fasting glucose in participants with obesity: results of a randomized, controlled, virtual clinical trial. Nutr Diabetes. 2021;11(1):6.

30. Sutton EF et al. Early time-restricted feeding improves insulin sensitivity, blood pressure, and oxidative stress even without weight loss in men with prediabetes. Cell Metab. 2018;27(6):1212-21.e3.

31. Beals JW, Kayser BD. When exercising for metabolic health, the work is never done, but HIIT will save you time. J Clin Endocrinol Metab. 2021;106:e365-e6.

32. Sabag A et al. Low-volume highintensity interval training for cardiometabolic health. J Physiol. 2021:DOI: 10.1113/JP281210.

33. RezkAllah SS, Takla MK. Effects of different dosages of interval training on glycemic control in people with prediabetes: a randomized controlled trial. Diabetes Spectr. 2019;32(2):12531.

34. De Nardi AT et al. High-intensity interval training versus continuous training on physiological and metabolic variables in prediabetes and Type 2 diabetes: a meta-analysis. Diabetes Res Clin Pract. 2018;137:14959.

35. Rowan CP et al. Aerobic exercise training modalities and prediabetes risk Reduction. Med Sci Sports Exerc. 2017:49(3):403-12.

36. Jung ME et al. High-intensity interval training as an efficacious alternative to moderate-intensity continuous training for adults with prediabetes. J Diabetes Res. 2015;2015:1-9.

37. Bourne JE et al. Brief exercise counseling and high-intensity interval training on physical activity adherence and cardiometabolic health in individuals at risk of type 2 diabetes: protocol for a randomized controlled trial. JMIR Res Protoc. 2019:8(3):e11226

38. Bean $C$ et al. "It's a life thing, not a few months thing": profiling patterns of the physical activity change process and associated strategies of women with prediabetes over 1 year. Can J Diabetes. 2020;44(8):701-10.

39. Francois ME et al. Carbohydraterestriction with high-intensity interval training: an optimal combination for treating metabolic diseases? Front Nutr. 2017;4:49

40. Srinivasan S, Florez JC. Therapeutic challenges in diabetes prevention: we have not found the "exercise pill". Clin Pharmacol Ther. 2015;98(2):162-9.

41. Echouffo-Tcheugui JB, Selvin E. Prediabetes and what it means: the epidemiological evidence. Annu Rev Public Health. 2021;42:59-77.

42. American Diabetes Association (ADA). 5. Prevention or delay of Type 2 diabetes: Standards of Medical Care in Diabetes-2018. Diabetes Care. 2018;41(Suppl 1):S51-4.

43. Centers for Disease Control and Prevention (CDD). Prediabetes - your chance to prevent type 2 diabetes. 2020. Available at: https://www.cdc. gov/diabetes/basics/prediabetes. html. Last accessed: 7 June 2021.

44. Bonora $E$ et al. Assessment of simple strategies for identifying undiagnosed diabetes and prediabetes in the general population. J Endocrino Invest. 2021:44(1):75-81.

45. Tuan C-Y et al. Usefulness of plasma glucose and insulin concentrations in identifying patients with insulin resistance. Am J Cardiol. 2003;92(5):606-10.

46. Sánchez-Ceinos J et al. miR-223 $3 p$ as a potential biomarker and player for adipose tissue dysfunction preceding type 2 diabetes onset. Mol Ther Nucleic Acids. 2021;23:1035-52.

47. Gar $\mathrm{C}$ et al. Serum and plasma amino acids as markers of prediabetes, insulin resistance, and incident diabetes. Crit Rev Clin Lab Sci. 2018;55(1):21-32 